\section{Comparação de dois programas computacionais utilizados na estimativa do consumo alimentar de crianças}

\section{Comparison of two nutrition software applications used to estimate food intake of children}

\section{Rosana Salles-Costa' \\ Marina M. L. Antunes ${ }^{2}$ \\ Melissa A. Mello ${ }^{3}$ \\ Rosely Sichieri ${ }^{4}$}

${ }^{1}$ Instituto de Nutrição Josué de Castro. Departamento de Nutrição Social e Aplicada - Universidade Federal do Rio de Janeiro

${ }^{2}$ Programa de Pós-Graduação em Nutrição - Instituto de Nutrição Josué de Castro - Universidade Federal do Rio de Janeiro

${ }^{3}$ Bolsista de Iniciação Científica (CNPq) - Curso de Graduação em Nutrição Instituto de Nutrição Josué de Castro - Universidade Federal do Rio de Janeiro

${ }^{4}$ Instituto de Medicina Social/Departamento de Epidemiologia - Universidade do Estado do Rio de Janeiro

Auxílio Financeiro: Conselho Nacional de Desenvolvimento Científico e Tecnológico - CNPq Edital MCT/MESA/CNPq/CT Agronegócio (processo no 503139/2003-3) e Instituto Nacional de Câncer (INCA/MS)

Correspondência: Rosana Salles-Costa. Instituto de Nutrição Josué de Castro/Departamento de Nutrição Social e Aplicada - Universidade Federal do Rio de Janeiro- UFRJ. Av. Brigadeiro Trompowsky s/n, CCS - Bloco J, $2^{\circ}$ andar - Illha do Fundão - Rio de Janeiro/RJ - CEP: 21941-590. Email: rosana_salles@terra.com.br

\section{Resumo}

Objetivo: Avaliar a concordância entre dois programas computacionais de análise do consumo alimentar de crianças de 6 a 30 meses. Materiais e métodos: Foram selecionadas 100 crianças de 6 a 30 meses de idade entre 407 crianças cujas mães responderam o recordatório de 24 horas em visitas domiciliares. Foram comparados: 1) as gramaturas das medidas caseiras dos 10 alimentos mais consumidos entre os dois programas e em relação a uma tabela de pesos e medidas nacional; 2) os valores médios do consumo de consumo de energia e nutrientes. A análise estatística foi feita através do coeficiente de correlação de Spearman e metodologia descrita por Bland \& Altman. Resultados: Foram observadas diferenças entre as gramaturas em relação à medida caseira correspondente para quase todos os alimentos selecionados. Comparando as médias de consumo entre os dois programas, observamos maior correlação entre o consumo de carboidratos $(r=0,81)$ e menor correlação para o consumo de zinco $(r=0,52)$. As médias de consumo de energia e de macronutrientes pelo programa Virtual Nutri foram superiores aos valores obtidos pelo NutWin, enquanto que para o consumo de micronutrientes observamos valores superiores na análise pelo program NutWin. O consumo de vitamina $\mathrm{C}$ e retinol revelou diferença reduzida entre os programas. Conclusão: Os programas selecionados para análise do consumo dietético infantil apresentam diferenças quanto às gramaturas utilizadas, que se refletem através das diferenças na estimava de energia e nutrientes consumidos.

Palavras-chave: Consumo alimentar. Alimentação infantil. Tabelas de composição de alimentos. 
Abstract

Objective: To evaluate the agreement between two nutrition software applications frequently used to estimate food intake in children from 6 to 30 months of age. Methods: One hundred children, from 6 to 30 months, were sampled from 407 children, whose mothers answered a 24 -hour recall. Interviews took place in the households. Sizes of portions for the 10 most frequently consumed foods were compared based on both software applications. Portion sizes in each software application were compared to the national table of portion sizes. Estimated energy intake and nutrients were also analyzed. The statistical analysis was conducted using the Spearman correlation coefficient and the Bland \& Altman method. Results: There were great differences between portion sizes in each software application in relation to the corresponding portion size in the national table for almost all foods selected. Comparing the mean intake between the two software applications, the greatest correlation was between carbohydrates $(\mathrm{r}=0.81)$ and the smallest correlation was observed for zinc $(\mathrm{r}=0.52)$. The mean energy intake and macronutrient intake by the Virtual Nutri program were higher than values based on the NutWin application. On the other hand, values of micronutrient intake were higher on the NutWin software. The two software applications differed slightly on the intakes of vitamin $C$ and retinol. Conclusion: The choice of software application may play an important role when estimating the food intake of children, both in relation to portion sizes and also because of food composition tables.

Keywords: Food intake. Children's food consumption. Food composition tables.

\section{Introdução}

A avaliação do consumo alimentar de uma população ou de grupos populacionais representa uma estratégia fundamental na área de pesquisa em nutrição e saúde possibilitando, entre outros aspectos, a organização de políticas públicas de prevenção e controle de distúrbios populacionais. É através destas informações que podemos estimar de forma mais precisa o quanto o consumo de energia ou de um determinado nutriente pode interferir na saúde de uma população. Deste modo encontra-se um grande número de pesquisas desenvolvidas há longa data, tanto no âmbito internacional como no nacional, com o objetivo de avaliar qualitativamente e quantitativamente o consumo de alimentos, dimensionar a adequação de nutrientes e relacionar a dieta à ausência de saúde ${ }^{1,2,3}$.

A estimativa do consumo de energia e nutrientes na infância é, particularmente, um desafio, devido à maior variabilidade de erros na mensuração da dieta relacionada às dificuldades de estimar o consumo alimentar diretamente com as crianças em função da dependência dos pais para relatar os alimentos consumidos por elas ${ }^{4}$. Olinto et al ${ }^{5}$ observaram uma tendência de superestimação no relato do consumo alimentar de crianças desnutridas obtidos através de entrevistas com as mães, utilizando o recordatório de 24 horas como instrumento de avaliação do consumo alimentar.

Cabe ressaltar que os erros na avaliação do consumo alimentar não estão relacionados apenas à metodologia escolhida para obtenção destas informações, mas também à conversão dos dados em quantidades de nutrientes, às tabelas e softwares de composição de alimentos. Embora a conversão da ingestão de alimentos a nutrientes e energia possa ser realizada por meio de análises químicas, freqüentemente são utilizadas as tabelas de composição de alimentos (TCAs) ${ }^{6}$.

A conversão das informações obtidas através do método escolhido para avalia- 
ção do consumo alimentar infantil através das TCAs é uma tarefa complexa. As TCAs podem apresentar variações que necessitam ser identificadas e controladas para a obtenção de estimativas de dieta o mais próximo possível do real consumo. Ribeiro et al $(2003)^{6}$ descrevem possíveis fatores que podem resultar em diferenças entre os dados avaliados. São eles:

- descrição incorreta de alimentos e/ou fontes de valores nutricionais;

- amostragem inadequada;

- utilização de métodos analíticos impróprios e inconsistência na terminologia utilizada para expressar certos nutrientes;

- variabilidade resultante de fatores genéticos, ambientais, de preparo e processamento.

A estes acrescentamos as possíveis diferenças quanto às porções utilizadas para apresentação dos alimentos, principalmente em TCAs informatizadas cuja base de dados é usualmente padronizada através de medidas caseiras.

A utilização de programas para avaliar o consumo alimentar infantil tem sido incentivada dado à praticidade de entrada de dados e obtenção das informações acerca do consumo alimentar. Entre eles destacam-se, no Brasil, os programas Virtual Nutri $^{7}$ e NutWin ${ }^{8}$.

O objetivo deste trabalho foi avaliar a concordância desses dois programas através da comparação entre as medidas caseiras utilizadas e os valores de energia, macro e micronutrientes (zinco, ferro, vitamina $\mathrm{C}$ e retinol) estimados para crianças.

\section{Material e Métodos}

Foram selecionados aleatoriamente 100 recordatórios de 24 horas dentre 407 registros com crianças na faixa etária de 6 a 30 meses, do estudo "Avaliação do estado nutricional, hábitos alimentares e insegurança alimentar no município de Duque de Caxias, Rio de Janeiro”, aprovado pelo Comitê de Ética e Pesquisa da Universidade Estadual do Rio de Janeiro em
1994, realizado no segundo distrito do município de Duque de Caxias, no Estado do Rio de Janeiro.

Inicialmente, os alimentos foram padronizados quanto à forma de apresentação para entrada nos bancos de dados (alimentos "in natura”, industrializados e preparações), bem como com relação à gramatura de algumas medidas caseiras ${ }^{9}$ para os casos de ausência de correspondência desta medida nos programas. Foram selecionados os programas Virtual Nutri ${ }^{7}$ e NutWin ${ }^{8}$ para análise comparativa do consumo alimentar das crianças através da comparação das medidas caseiras (gramatura) dos 10 alimentos mais consumidos, da freqüência de consumo total dos 1005 alimentos citados nos recordatórios de 24 hs, e consumo médio de energia (kcal), de macronutrientes (proteínas, carboidratos e lipídeos - em gramas) e de micronutrientes (ferro, zinco, vitamina $\mathrm{C}$ e retinol- $\mathrm{mg}$ ).

Foram comparadas as gramaturas das medidas caseiras dos dois programas (Virtual Nutri - NutWin), contrastando os valores com uma tabela padrão de pesos e medidas caseiras ${ }^{9}$. Em seguida, utilizou-se o coeficiente de correlação de Spearman e, para a identificação de padrões sistemáticos entre os valores aferidos entre os programas, foi utilizada a metodologia recomendados por Bland e Altman $(1986)^{10}$, na qual, para o consumo médio estimado de nutrientes e energia, as diferenças entre os dois métodos (por exemplo, Virtual

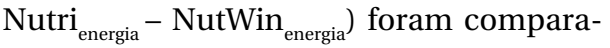
das graficamente com as médias dos dois métodos ((Virtual Nutri ${ }_{\text {energia }}+$ NutWin $_{\text {energia }}$ ) $\div 2$ ). Uma linha horizontal representa o ponto de concordância entre os programas (zero); quanto mais próximos do eixo de concordância estiverem os pontos, maior a concordância entre os programas. Para análise foi utilizado o programa Stata, versão $9.0^{11}$.

\section{Resultados}

Na Tabela 1, as opções de medida caseira e a gramatura correspondentes de 
Tabela 1 - Comparação entre a gramatura das medidas caseiras dos alimentos mais consumidos, estimadas através de dois programas de avaliação do consumo alimentar com os valores de uma tabela de referência de pesos e medidas.

Table 1 - Comparison between portion sizes of most frequently consumed foods, estimated by two nutrition software applications with a reference table of portion sizes.

\begin{tabular}{|c|c|c|c|c|c|c|}
\hline Alimentos & Medida Caseira & $\begin{array}{l}\text { Virtual Nutri } \\
\text { (gramas) }\end{array}$ & $\begin{array}{l}\text { NutWin } \\
\text { (gramas) }\end{array}$ & $\begin{array}{c}\text { Diferença entre } \\
\text { as medidas } \\
\text { (Virtual Nutri- } \\
\text { NutWin) }\end{array}$ & $\begin{array}{l}\text { Medida cheia/ } \\
\text { unidade* } \\
\text { (gramas) }\end{array}$ & $\begin{array}{l}\text { Medida } \\
\text { rasa* }^{*} \\
\text { (gramas) }\end{array}$ \\
\hline \multirow[t]{4}{*}{ Açúcar refinado } & colher de sopa & 28,0 & 15,0 & 13,0 & 24,0 & 15,0 \\
\hline & colher de café & 2,6 & 2,0 & 0,6 & 2,0 & 1,0 \\
\hline & colher de chá & 4,4 & 3,5 & 0,9 & 5,0 & 3,0 \\
\hline & colher de sobremesa & 16,0 & 10,0 & 6,0 & 16,0 & 9,0 \\
\hline \multirow[t]{4}{*}{ Arroz } & colher de sopa & 31,0 & 20,0 & 11,0 & 25,0 & 15,0 \\
\hline & colher de servir & 50,0 & 45,0 & 5,0 & 45,0 & 30,0 \\
\hline & colher de sobremesa & 20,0 & $* *$ & - & $* *$ & $* *$ \\
\hline & escumadeira & 106,0 & 85,0 & 21,0 & 85,0 & 60,0 \\
\hline \multirow[t]{2}{*}{ Leite em pó } & colher de sopa & 15,0 & 8,0 & 7,0 & 16,0 & 8,0 \\
\hline & colher de sobremesa & 8,8 & 5,0 & 3,8 & 9,0 & 5,0 \\
\hline \multirow[t]{5}{*}{ Feijão (c/ caroço) } & colher de sopa & 26,2 & 18,0 & 8,2 & 17,0 & $* *$ \\
\hline & colher de sobremesa & 23,0 & $* *$ & - & $* *$ & $* *$ \\
\hline & colher de servir & 70,0 & $* *$ & - & 35,0 & $* *$ \\
\hline & concha média & 86,0 & 117,0 & $-31,0$ & 140,0 & 80,0 \\
\hline & concha pequena & $* *$ & 60,0 & - & 65,0 & 40,0 \\
\hline \multirow[t]{4}{*}{ Feijão (só caldo) } & colher de sopa & 11,0 & $* *$ & - & $* *$ & $* *$ \\
\hline & colher de sobremesa & 9,3 & $* *$ & - & $* *$ & $* *$ \\
\hline & concha média & 82,0 & 80,0 & 2,0 & $* *$ & $* *$ \\
\hline & concha pequena & $* *$ & 65,0 & - & $* *$ & $* *$ \\
\hline \multirow[t]{2}{*}{ Banana } & unidade média & 53,0 & 70,0 & $-17,0$ & 40,0 & $* *$ \\
\hline & unidade pequena & $* *$ & 40,0 & - & 30,0 & $* *$ \\
\hline \multirow[t]{4}{*}{ Batata cozida } & fatia & 5,0 & 14,0 & $-9,0$ & $* *$ & $* *$ \\
\hline & unidade pequena & 31,0 & 70,0 & $-39,0$ & 70,0 & $* *$ \\
\hline & unidade média & $* *$ & 136,0 & - & 140,0 & $* *$ \\
\hline & colher de sopa & $* *$ & 16,0 & - & 30,0 & $* *$ \\
\hline \multirow[t]{3}{*}{ Leite fluido } & copo tipo requeijão & 208,0 & 250,0 & $-42,0$ & 240,0 & $* *$ \\
\hline & copo americano & $* *$ & 150,0 & - & 165,0 & $* *$ \\
\hline & xícara de chá & $* *$ & 160,0 & - & $* *$ & $* *$ \\
\hline \multirow[t]{4}{*}{ Macarrão } & colher de sopa & 30,0 & $* *$ & - & 25,0 & $* *$ \\
\hline & colher de sobremesa & 25,0 & $* *$ & - & $* *$ & $* *$ \\
\hline & escumadeira & 70,0 & $* *$ & - & 110,0 & 75,0 \\
\hline & pegador & 110,0 & 60,0 & 50,0 & 110,0 & $* *$ \\
\hline Pão francês & unidade & 50,0 & 50,0 & 0,0 & 50,0 & $* *$ \\
\hline \multirow[t]{2}{*}{ Mucilon arroz } & colher de sopa & 8,0 & 8,0 & 0,0 & 9,0 & 5,0 \\
\hline & colher de sobremesa & 5,0 & $* *$ & - & 5,0 & 3,0 \\
\hline Biscoito maizena & unidade & 5,0 & 5,0 & 0,0 & 5,0 & $* *$ \\
\hline \multirow[t]{2}{*}{ Cenoura cozida } & colher de sopa & 25,0 & 13,0 & 12,0 & 25,0 & 15,0 \\
\hline & unidade & 82,0 & 75,0 & 7,0 & 135,0 & 55,0 \\
\hline \multirow[t]{2}{*}{ Cremogema } & colher de sopa & 25,0 & 11,0 & 14,0 & 20,0 & 10,0 \\
\hline & colher de sobremesa & 16,0 & $* *$ & - & 13,0 & 7,0 \\
\hline Farinha Láctea & colher de sopa & 7,5 & 7,0 & 0,5 & 20,0 & 10,0 \\
\hline
\end{tabular}

*Gramaturas obtidas na tabela de pesos e medidas de referência/Portion sizes from a reference weights and portion sizes table

**Valores não encontrados na tabela de pesos e medidas utilizada/Values not found on reference weights and portion sizes table 
cada programa foram comparadas entre si e com os valores equivalentes de uma tabela de pesos e medidas caseiras $^{9}$ dos 10 alimentos mais citados no consumo alimentar das crianças. Observou-se diferenças entre os programas com relação às gramaturas correspondentes da mesma medida caseira utilizada, exceto para o pão francês, o Mucilon e o biscoito maizena. Para a relação de alimentos encontrada, as medidas caseiras dos dois programas não utilizam como padrão a correspondência de "medida cheia" ou "medida rasa", o que acentuou a diferença de gramatura entre eles, como observamos para o açúcar, o arroz, o leite em pó e as farinhas infantis (Cremogema, Farinha Láctea, Mucilon e outros), intensificando a diferença entre os valores encontrados nos programas e a tabela de referência utilizada.

Quando avaliamos a correlação entre os valores de energia, macro e micronutrientes estimados pelos dois programas, o consumo de carboidratos obteve a maior correlação ( $r=0,81)$ e o consumo de zinco foi menos correlacionado $(r=0,52)$ (Tabela 2).

Utilizando o procedimento descrito por Bland \& Altman, observa-se na Tabela 3 que existe uma tendência das médias de consumo de energia, carboidratos, lipídeos e retinol, pelo programa Virtual $\mathrm{Nu}$ $\operatorname{tri}^{7}$, serem significativamente superiores ao valores obtidos pelo NutWin ${ }^{8}$, enquanto que para o consumo de ferro, vitamina $\mathrm{C} \mathrm{e}$ zinco observamos uma tendência de valores superiores na análise pelo programa NutWin ${ }^{8}$. Entretanto, as diferenças não foram significativas quando avaliamos os intervalos de confianca (IC95\%).

Tabela 2 - Coeficiente de Correlação de Spearman entre os valores de energia, macro e micronutrientes obtidos através da comparação do consumo alimentar de crianças estimados pelos programas Virtual Nutri e NutWin.

Table 2 - Spearman Coefficients correlation between energy, macro and micronutrients estimated through the comparison of children food intake estimated by the Virtual Nutri and NutWin software.

\begin{tabular}{lc}
\hline $\begin{array}{l}\text { Energia e } \\
\text { nutrientes }\end{array}$ & $\begin{array}{c}\text { Coeficiente } \\
\text { de Correlação }\end{array}$ \\
\hline Energia & $0,79^{*}$ \\
Carboidratos & $0,81^{*}$ \\
Proteínas & $0,71^{*}$ \\
Lipídeos & $0,80^{*}$ \\
Zinco & $0,52^{*}$ \\
Vitamina C & $0,70^{*}$ \\
Ferro & $0,66^{*}$ \\
Retinol & $0,63^{*}$
\end{tabular}

* Valores estatisticamente significantes ( $p$ valor $<0,0000)$; Statiscally significant values ( $p$ value $<0.000$ )

Tabela 3 - Diferença entre as médias de consumo de energia, macro e micronutrientes, e seus respectivos valores de intervalos de confiança (IC 95\%) entre dois programas de avaliação do consumo alimentar (Virtual Nutri- NutWin).

Table 3 - Differences among the mean of energy intake, macro and micronutrients intake and confidence intervals (95\% IC) between two nutrient software (Virtual Nutri- NutWin).

\begin{tabular}{lccc}
\hline Energia e Nutrientes & $\begin{array}{c}\text { Diferença entre } \\
\text { as médias }\end{array}$ & Desvio Padrão & IC 95\% \\
\hline Energia (Kcal) & 224 & 429 & 140 a 309 \\
Carboidratos (g) & 17,6 & 65,5 & 4,6 a 30,5 \\
Proteínas (g) & 2,6 & 24,7 & $-2,6$ a 7,4 \\
Lipídeos (g) & 10,3 & 13,4 & 7,6 a 13,0 \\
Ferro (mg) & $-0,5$ & 4,5 & $-15,5$ a 16,9 \\
Vitamina C (mg) & $-4,5$ & 54,8 & $-15,3$ a 6,3 \\
Zinco (mg) & $-0,5$ & 3,5 & $-1,4$ a 0,3 \\
Retinol & 453 & 2128 & 35,6 a 871,9 \\
\hline
\end{tabular}


A análise gráfica permite visualizar as diferenças entre os programas, já que, exceto para a vitamina $\mathrm{C}$ e retinol, a maioria dos pontos situou-se distante da linha horizontal (eixo 0). Comparando-se a concentração dos pontos acima e abaixo desta linha confirmamos a tendência a valores inferiores do consumo de energia e macronutrientes pelo programa NutWin ${ }^{8}$ e, por sua vez, estimativa de valores superiores do consumo de zinco e ferro.

\section{Discussão}

A escolha da tabela de composição de alimentos é uma tarefa importante para a análise de inquéritos dietéticos, principalmente no nosso país, dadas as restrições de infomações de análises de alimentos. A padronização das medidas caseiras e porções consumidas consiste em outra fonte de erro importante que pode interferir diretamente na estimativa do consumo real de alimentos.

Os valores das porções utilizadas em cada programa analisado com a tabela de referência escolhida indicam variações importantes em ítens de consumo muito freqüente; por exemplo, quando a medida caseira utilizada para o consumo de açúcar refinado era a colher de sopa, a gramatura da medida rasa do programa Virtual Nutri ${ }^{7}$ era correspondente a quase o dobro da medida cheia do outro programa. Quando comparamos as diferenças das gramaturas entre os dois programas, foi possível constatar que o Programa Virtual Nutri ${ }^{7}$ tende a apresentar gramaturas maiores do que o programa NutWin ${ }^{8}$, com relação aos alimentos e medidas caseiras utilizadas. Entretanto, não podemos afirmar que este programa tende sempre a super estimar a gramatura dos alimentos, quando comparamos as diferenças encontradas com a gramatura da tabela de referência ${ }^{9}$

Dos três alimentos que não apresentaram diferença na gramatura, dois deles (pão francês e biscoito maisena) são alimentos cuja gramatura é padronizada pelos fabricantes.
As diferenças encontradas interferiram principalmente no consumo médio de energia, sendo os carboidratos os principais responsáveis pelas diferenças; o valor do desvio padrão de 65,5 gramas, observado para a diferença da média de consumo, equivale a $262 \mathrm{kcal}$. O consumo de lipídios também interferiu de forma importante na média de energia consumida.

Neste estudo, foi possível observar diferenças importantes entre os programas selecionados quanto à gramatura em relação aos alimentos mais consumidos obtidos através do R-24 na amostra de crianças avaliadas.

Com relação aos micronutrientes avaliados, existem variações quanto à variabilidade de alimentos avaliados nos dois programas, dadas as diferenças das bases de dados utilizadas. O programa NutWin ${ }^{8}$ tem como base de dados a tabela norteamericana de composição de alimentos (USDA) ${ }^{12}$ e a de Philippi (2002) ${ }^{13}$, e enquanto o Virtual Nutri ${ }^{7}$ utiliza compilações destas informações de tabelas nacionais e internacionais $^{13}$. O esforço para uma tabela nacional de análise química dos alimentos, ainda ausente no nosso país, minimizaria esse problema.

Entre os micronutrientes avaliados no presente estudo, a vitamina $\mathrm{C}$ e o retinol foram os elementos que apresentaram distribuição dos valores mais próxima do zero, quando comparamos os valores obtidos entre as diferenças das médias e as médias entre os programas (Figura 1). Estes resultados são corroborados pela boa correlação entre as médias (Tabela 2), ou seja, a composição das tabelas para estes micronutrientes apresenta pouca variabilidade.

A diferença encontrada na composição de micronutrientes pode influenciar diretamente na determinação do percentual de crianças com défict no consumo. Como exemplo, se avaliarmos o consumo de zinco entre os R-24 avaliados, tendo como base as recomendações internacionais (DRIs) ${ }^{14}$ de $3 \mathrm{mg} /$ dia para crianças na faixa etária de estudo, pelo programa Virtual Nutri teríamos um total de $40,6 \%(n=41)$ da popula- 

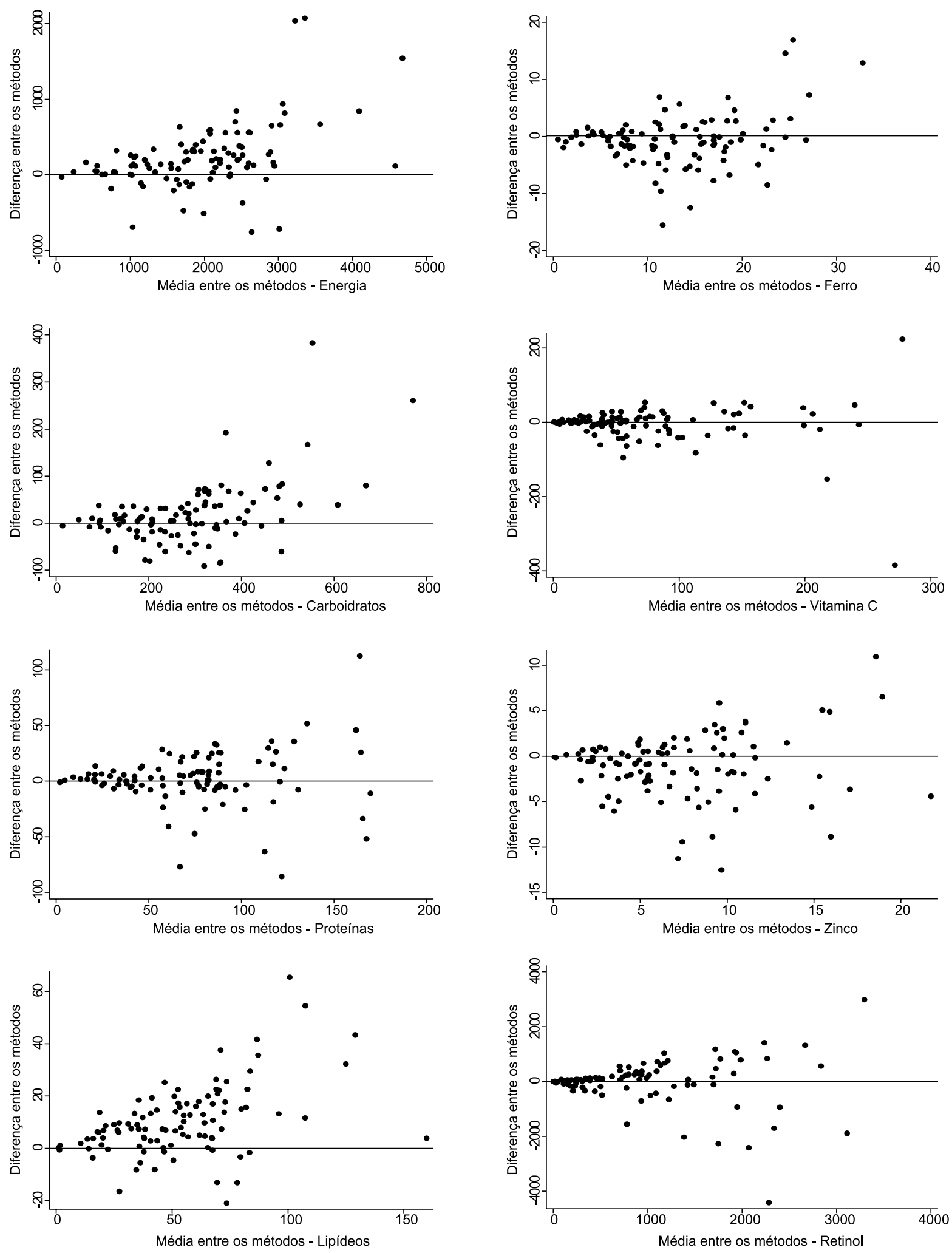

Figura 1 - Relação entre a diferença e as médias dos programas utilizados com relação ao consumo dietético de energia e nutrientes.

Figure 1 - Relation between the difference and the means of the nutrient software applications used to estimate energy intake and nutrient intake. 
ção estudada com déficit de consumo, enquanto que pelo programa NutWin ${ }^{8}$, para a mesma a amostra, teríamos $23,8 \% \quad(n=24)$ das crianças com déficit no consumo. Realizando o mesmo procedimento para as recomendações de ferro (DRI=7mg/dia), a diferença entre eles é menor; 47,5\% (n=48) e $40,8 \%(n=41)$ das crianças apresentaram consumo inadequado para ambos os programas, Virtual Nutri ${ }^{7}$ e NutWin ${ }^{8}$. Apesar da diferença entre as freqüências não ter sido significativa ( $p>0,05)$, observamos que o segundo programa tende a apresentar um maior número de alimentos avaliados para a composição de zinco.

Cabe ressaltar que esta limitação pode ser minimizada nos dois programas através do acesso à base de dados que permite para os casos de ausência de análise daquele nutriente a correção da informação utilizando outra fonte de dados. Ou seja, o acesso a outras tabelas de composição, rótulos, embalagens ou mesmo contato com os fabricantes pode minizar a ausência da informação quando presente.

Se considerarmos que alguns autores observaram uma superestimação no consumo alimentar de crianças, avaliado a partir do R-24 horas realizado com suas mães ${ }^{5,15-17}$, os resultados observados sugerem que a utilização do programa NutWin' talvez seja mais adequada para estimar o consumo alimentar de crianças. Entretanto, deve ser ressaltado que os dois programas permitem a entrada de dados dos alimentos na opção "em gramas” para todos os ítens aqui analisados, o que minimiza as diferenças entre a média de consumo para energia e de macronutrientes. Sugere-se padronizar antes da digitação de dados, as gramaturas dos alimentos consumidos com base nos dados da pesquisa.

\section{Conclusão}

Concluí-se que os programas selecionados para análise do consumo dietético infantil apresentam diferenças quanto às gramaturas utilizadas, com reflexos importantes na estimativa de energia e nutrientes do consumo alimentar infantil, como também na análise de micronutrientes.

\section{Referências}

1. Livingstone MBE, Robson PJ, Wallace JM. Issues in dietary intake assessment of children and adolescents. $\mathrm{Br}$ J Nutr 2004; 92(2): S213-S222.

2. SSawaya AL, Martins P, Hoffman D, Roberts SB. The link between childhood undernutrition and risk of chronic diseases in adulthood: a case study of Brazil Nutr Rev 2003; 61 (5 Pt 1): 168-75.

3. N, Yang SJ, Baranowski T, Zakeri I, Berenson G. Eating patterns and obesity in children. The Bogalusa Heart Study. Am J Prev Med 2003; 25(1): 9-16.

4. Livingstone MBE, Robson PJ. Measurement of dietary intake in children. Proc Nutr Soc 2000; 9: 279-93.

5. Olinto MT, Victora CG, Barros FC, Gigante DP. Twentyfour-hour recall overestimates the dietary intake of malnourished children. J Nutrition 1995, 125(4): 880-4.

6. Ribeiro T, Moraes TB, Colugnati FAB, Sigulem DM. Tabelas de composição química dos alimentos: análise comparativa com resultados laboratoriais. Rev Saúde Pública 2003; 37(2):216-25.
7. Philippi ST, Szarfarc SC, Latterza AR. Virtual Nutri [software]. Versão 1.0 for Windows. Departamento de Nutrição/Faculdade de Saúde Pública. Universidade de São Paulo; 1996.

8. Programa de Apoio a Nutrição - NutWin [software]. Departamento de Informática em Saúde. Universidade Federal de São Paulo; 2005.

9. Pinheiro ABV, Lacerda EMA, Benzecky EG, Gomes MCS, Costa VM. Tabela para avaliação de consumo alimentar em medidas caseiras. 5ª ed. Ed. Atheneu: São Paulo; 2004.

10. Bland JM, Altman DG. Statistical methods for assessing agreement between two methods of clinical measurement. Lancet 1986; 8: 307-10.

11. STATA. Stata Corporation: version 9.0. Texas: College Station, USA; 2006.

12. US Department of Agricultural. Agricultural Research Service. USDA nutrient database for standard reference release 11, 1998. Disponível em URL: http.// www.nal.usda.gov/fnic/foodcomp. Acessado em 2 de março de 2006. 
13. Philippi ST. Tabela de composição de alimentos: suporte para decisão nutricional. Editora Coronário: São Paulo; 2002.

14. Institute of Medicine. National Research Council. Dietary Reference Intakes for energy, carbohydrate, fiber, fat, fatty acids, cholesterol, protein and amino acids. Washington (DC): National Academy Press; 2002.

15. Black AP, Salles-Costa R. Análise do recordatório de 24 horas como método de avaliação do consumo alimentar infantil e sua associação com as concentrações séricas de ferro e zinco e déficit ponderal em crianças residentes em uma comunidade de baixa renda. Dissertação de Mestrado. Programa de Pós-Graduação em Nutrição Instituto de Nutrição Josué de Castro/UFRJ, Rio de Janeiro, 2006, 87p.
16. Montgomery C, Reilly JJ, Jackson DM, Kelly LA, Slater C, Paton JY, Grant S. Validation of energy intake by 24 -hour multiple pass recall: comparison with total energy expenditure in children aged 5-7 years. BrJ Nutr 2005; 93(5): 671-6.

17. Kaskoun MC, Johnson RK, Goran MI. Comparison of energy intake by semiquantitative food-frequency questionnaire with total energy expenditure by the doubly labeled water method in young children. Am J Clin Nutr 1994; 60(1).

Recebido em: 05/07/06 Versão final reapresentada em: 09/03/07 Aprovado em: 17/04/07 